Das Forum Managed Care bietet uns nachfolgend eine hervorragende Übersicht über die aktuelle Situation in Bezug auf die Versorgungsnetze und das zunehmend als «integrierte Versorgung» bezeichnete System.

Die in diesem Dokument präsentierte «Sicht der Ärztenetze» entspricht wie die meisten anderen Punkte der Zusammenfassung der Haltung der FMH zu diesem Thema.

Für die FMH ist auch klar, dass die Wahl zwischen der «integrierten Versorgung" und dem klassischen System unbedingt beibehalten und für alle, d.h. Patientinnen und Patienten sowie Ärztinnen und Ärzte, frei bleiben muss. Die notwendigen Anreize müssen vor dem Hintergrund dieser Anforderung gestaltet werden. Das Bestehen eines Vertrags zwischen der betroffenen Ärztegruppe und einem oder mehreren Versicherern ist auch für uns eine Grundvoraussetzung, und die «Listenmodelle» sind wegen ihrer Untauglichkeit klar zu bekämpfen.

«Managed-Care»-Verträge mit einzelnen Ärzten sind genauso unsinnig (Wie soll eine einzelne Person ein Netz oder eine Behandlungskette bilden?!) und nicht annehmbar. Wenn in einer Region kein Netz aufgebaut werden kann, könnte allenfalls die kürzlich von den kantonalen Gesundheitsdirektoren vorgeschlagene Lösung eine mögliche Alternative darstellen und wäre näher zu prüfen.

Die FMH dankt dem Forum Managed Care für dieses ausgezeichnete Übersichtsdokument und freut sich über dessen Veröffentlichung.

\title{
Orientierungspapier «Integrierte Versorgung in der Schweiz»
}

Forum Managed Care

\footnotetext{
* In gewissen Regionen (z. B. im Kanton Thurgau) können Versicherte bereits heute auch einen Spezialisten als primäre Anlaufstelle bezeichnen.
}

Managed Care bezeichnet die Summe aller Steuerungs- und Integrationsinstrumente im Gesundheitswesen mit dem Ziel, eine bedürfnisgerechte, qualitätsbasierte und kosteneffektive medizinische Versorgung zu fördern. Der Begriff «Managed Care» wird allerdings kaum mehr benutzt, vielmehr spricht man von «integrierter Versorgung». International sind eine Vielzahl solcher Steuerungs- und Integrationsansätze eingeführt worden, beispielsweise integrierte Organisationsformen (Hausarztmodelle, Ärztenetze, $\mathrm{HMO} /$ Gesundheitszentren) oder Integrationsinstrumente (Gatekeeping, Guidelines, Case Management, Disease Management, Demand Management/Telefontriage).

\section{Integrierte Versorgung als Versicherungsform} Mit Inkrafttreten des KVG am 1. Januar 1996 wurde es möglich, alternative Versicherungsmodelle (AVM) anzubieten. Ausgehend vom Subsidiaritätsprinzip unseres liberalen Staates können seither die Vertragspartner, also Ärzte und Versicherer, solche Modelle entwickeln und anbieten. Heute können Versicherte innerhalb der regulären Krankenversicherung zwischen verschie- denen AVM wählen - häufig auch Managed-CareModelle genannt - und sich als Patienten in Ärztenetzen/HMO behandeln und betreuen lassen.

Allen AVM gemeinsam ist das Prinzip des Gatekeeping: Die versicherte Person verpflichtet sich, bei gesundheitlichen Beschwerden immer den gleichen «Eingang» ins Gesundheitswesen zu nehmen. Dies kann der Hausarzt sein, ein Gesundheitszentrum (HMO) oder aber ein medizinisches Callcenter. Spezialisierte oder stationäre Behandlungen sind nur nach Überweisung durch den Gatekeeper oder Care Manager möglich («eingeschränkte Arztwahl»). ${ }^{*}$ Im Gegenzug erhalten die Versicherten einen Prämienrabatt. Ausgenommen von dieser Verpflichtung sind Notfälle. Sonderregelungen gibt es meist für den Besuch eines Frauen-, Kinder- oder Augenarztes.

In gewissen Regionen hat sich bereits über ein Drittel der Versicherten (z. B. Kanton Thurgau) für ein alternatives Versicherungsmodell entschieden.

\section{Modelle der integrierten Versorgung}

In der Schweiz haben sich verschiedene Modelle der integrierten Versorgung entwickelt:
Korrespondenz: Forum Managed Care Pflanzschulstrasse 3 CH-8400 Winterthur Tel. 0522350553

info@fmc.ch

www.fmc.ch

\footnotetext{
Charakteristiken eines Ärztenetzes (angelehnt an die Definition von med-swiss.net)

- Ärztebasiert: Werden von Leistungserbringern gebildet und dienen der Gesundheitsversorgung

- Verbindlichkeit: Vertraglich geregelte Zusammenarbeit unter sich, mit netzfremden Leistungserbringern und Kostenträgern

- Einheitliche Philosophie: Handeln auf der Basis von vereinbarten Behandlungsprozessen, unternehmerischen Organisationsstrukturen und einer gemeinsamen Betreuungskultur

- Care Management: Steuern die Gesundheitsleistungen so weit wie möglich aus einer Hand

- Bedürfnisorientiert: Richten sich konsequent auf die Bedürfnisse der Patientinnen und $\mathrm{Pa}$ tienten aus

- Qualitätsorientiert: Setzen sich für eine hohe Versorgungsqualität ein

- Kostenbewusst: Gehen mit den vorhandenen Mitteln haushälterisch um
} 
Vertragsmodelle (Ärztenetze, HMO, Medizinische Callcenter)

In Ärztenetzen bilden die Hausärzte eine Gruppe, die mit der Krankenkasse einen speziellen Vertrag hat. Darin verpflichten sich die Netze, ihre Ärzte konsequent weiterzubilden. Dies geschieht primär in sogenannten Qualitätszirkeln. Hier treffen sich die Ärztinnen und Ärzte regelmässig, um komplexe Einzelfälle $\mathrm{zu}$ besprechen, mögliche Fehlerquellen bei Behandlungen zu ermitteln oder neue Therapien zu bewerten. Weiter verpflichten sich die Netze, die Steuerung systematisch zu kontrollieren. Die Ärztenetze übernehmen als Kollektiv häufig eine finanzielle Mitverantwortung (Budgetmitverantwortung in Form eines Bonus-Malus-Systems oder Capitation-Vertrages). Sie erhalten eine Vergütung für den zusätzlichen Aufwand für die Steuerung und Qualitätsarbeit. Analoge Anforderungen gelten für $\mathrm{HMO} / \mathrm{Gesundheitszentren.}$ Die meisten von ihnen gehören Krankenversicherern, und die Ärzte sind angestellt. Die übrigen sind im Besitz der Ärzte, die dort arbeiten.

In der Schweiz gibt es rund 90 Ärztenetze und HMO. Knapp die Hälfte aller Grundversorgerinnen und Grundversorger engagieren sich in Ärztenetzen. Zwei von drei solcher Netze haben mit den Krankenversicherern eine Budgetmitverantwortung vereinbart.

Bei Modellen mit einem medizinischen Callcenter haben die Versicherten die Pflicht, sich bei jedem Gesundheitsproblem zuerst telefonisch beraten $\mathrm{zu}$ lassen. Dabei geben Fachpersonen Auskünfte und Verhaltensempfehlungen ab oder aber sie weisen die Patienten an einen Arzt oder ein Spital weiter. Bei diesen Modellen besteht ein spezieller Vertrag zwischen Versicherer und Callcenter. Die Steuerung ist eher punktuell und beschränkt sich heute in der Regel auf das Telefonat. Weitergehende Behandlungs- und Betreuungsangebote für bestimmte Patienten durch die Telemedizin sind aber möglich und werden teilweise auch angeboten.

\section{Nichtvertragsmodelle (Listenmodelle, Lightmodelle)}

Nichtvertragsmodelle kennen keine besondere vertragliche Regelung zwischen Versicherern und Ärzten. Diese Modelle sind Versicherungsprodukte, bei denen die Versicherer Leistungserbringerlisten definieren, weshalb sie auch «Listenmodelle» genannt werden. Die Listenmodelle basieren ebenfalls auf der eingeschränkten Wahl des Leistungserbringers. Sie sind aus folgenden Gründen umstritten:

- Ärztenetze und Ärzte bezeichnen Listenmodelle wegen der fehlenden vertraglichen Partnerschaft auch als Pseudo- oder Lightmodelle. Strittige Punkte sind die von den Versicherern definierten Leistungserbringerlisten sowie die fehlende Verbindlichkeit; dadurch werde nur der Risikoselektion Vorschub geleistet und die Entwicklung von integrierter Versorgung stark behindert.

- Versicherer sind überzeugt, dass Listenmodelle in Regionen ohne Vertragsmodelle (Ärztenetze/
HMO) sinnvoll sind und als Möglichkeit zur Kosteneinsparung angeboten werden sollen. Das Verhalten der Versicherten soll daher auch in diesen Modellen belohnt werden. Dass Listenmodelle die Entwicklung der integrierten Versorgung behindern, bestreiten die Versicherer aufgrund des zunehmenden Marktanteils aller AVM.

\section{Integrierte Modelle in Entwicklung}

Das zentrale Steuerungsinstrument der heutigen Modelle ist das Gatekeeping. Durch die Koordination der Überweisungen an spezialisierte bzw. stationäre Betreuende sollen die Gatekeeper Doppelspurigkeiten und unnötige Behandlungen vermeiden. Das funktioniert bei Bagatellerkrankungen und gewissen Krankheiten einwandfrei.

Integrierte Versorgung und Steuerung machen vor allem dort Sinn, wo viele und unterschiedlichste Behandlungen und/oder Betreuungen aufeinander abgestimmt und koordiniert werden sollten. Dies ist insbesondere bei komplexen, chronischen Krankheiten und bei Langzeitbetreuung der Fall. Hier verspricht eine integrierte Versorgung die Optimierung der Behandlungsqualität, die Gewährleistung der Versorgungssicherheit und die Steigerung der Kosteneffektivität. Gerade diese Patientinnen und Patienten sollten Anreize haben, dass sie von einer integrierten Versorgung angezogen werden. Deshalb wären hier neben Prämienrabatten auch Reduktionen der Kostenbeteiligung als Anreiz sinnvoll.

\section{Integrierte Versorgung aus Sicht der wichtigsten Akteure}

Angesichts der unterschiedlichen Funktionen und Interessen der Akteure darf es nicht erstaunen, dass auch deren Vorstellungen an (gesetzliche) Grundlagen und Anreize zu Weiterentwicklungen in Teilaspekten (noch) gegensätzlich sind. Die Positionen dazu:

\section{Sicht der Ärztenetze}

1. Grundlage der Ärztenetze ist ein Vertrag zwischen Versicherern und dem Ärzte-/Versorgungsnetz (Gruppe von Leistungserbringern); Verträge mit einzelnen Ärzten sollten die Ausnahme sein. In einem Vertrag mit einem Netzwerk sind die Modalitäten der Zusammenarbeit, das Leistungsangebot (eine allfällige Übernahme von Nichtpflichtleistungen) und dessen Vergütung geregelt. Zudem werden eine Budgetmitverantwortung, eine Risikobeteiligung sowie Qualitätssicherungsmassnahmen und deren Vergütung festgelegt. Im Ärztenetz erzielte Einsparungen (inkl. logistische Einsparungen) sollen im System des Versorgungsvertrags verbleiben.

2. In den Ärztenetzen ist ein Betreuungsarzt oder ein ärztlich geführtes Betreuungsteam erste Anlaufstelle für den Patienten. Diese koordinieren alle notwendigen Abklärungen und Behandlungen und stellen die Beziehungen und Koordination der an der Behandlung des Patienten beteiligten 
Leistungserbringer sicher. Die Ärztenetze organisieren sich autonom. Das Netzwerk regelt die Zusammenarbeit mit Spezialisten und weiteren Leistungserbringern, namentlich den Spitälern. Die Ärztenetze definieren und unterhalten ein Qualitätskonzept mit entsprechenden Qualitätsindikatoren und weisen die erreichten Ergebnisse aus.

3. Versicherte und Leistungserbringer sollen sowohl den Beitritt in ein Modell der integrierten Versorgung bzw. ein Ärztenetz frei wählen können wie auch den Austritt. Längerfristige Verträge für Versicherte (länger als ein Jahr) sollen möglich sein. Versicherte in integrierten Modellen sollen gegenüber konventionell Versicherten finanziell begünstigt werden (Prämienrabatte, differenzierter Selbstbehalt).

\section{Sicht der Versicherer}

1. Bei alternativen Versicherungsmodellen entscheiden sich die Versicherten freiwillig, die Wahl der Leistungserbringer einzuschränken. Dadurch werden eine bessere Koordination der Behandlung und nachgewiesenermassen tiefere Behandlungskosten möglich. Die tieferen Kosten sollen in Form von tieferen Prämien und eventuell tieferen Selbstbehalten an die Versicherten weitergegeben werden. Die Ärztenetze sollen dabei Budgetverantwortung übernehmen und eine Vergütung für die Steuerung erhalten.

2. Die bestehenden gesetzlichen Regelungen ermöglichen eine grosse Vielfalt alternativer Versicherungsmodelle. Diese soll nicht eingeschränkt werden. Leistungserbringer und Versicherer können besondere Modelle frei vereinbaren und auf die jeweiligen Gegebenheiten und Bedürfnisse der Versicherten ausrichten. Insbesondere ist auf einen speziellen Vertragszwang zu verzichten, da dieser Flexibilität, Einsparpotential und geografische Ausdehnung hemmt. Zudem sollten Mehrjahresverträge und Aufnahme von Nichtpflichtleistungen möglich sein.

3. Ein differenzierterer Risikoausgleich vermindert Risikoselektion durch die Versicherer (beispielsweise durch Billigkassen) und fördert integrierte Versorgung mit Blick auf Qualität und Wirtschaftlichkeit. Ob der ab 2012 geplante verfeinerte Risikoausgleich deshalb noch weiterentwickelt werden soll, muss aufgrund zu erarbeitender wissenschaftlicher Grundlagen entschieden werden.

4. Die Palette der möglichen Anreize soll derart erweitert werden, dass integrierte Versorgung bzw. alternative Versicherungsmodelle für kranke Versicherte attraktiver werden. Das heisst, neben Prämienrabatten sind auch differenzierte Kostenbeteiligungen (differenzierter Selbstbehalt) möglich.

\section{Sicht der Patienten}

1. Die bisherigen Erfahrungen mit den alternativen Versicherungsmodellen zeigen, dass Wahlmöglich- keiten für Versicherte und Patienten bedeutsam sind. Versicherte und Patienten sollen deshalb innerhalb der Grundversicherung zwischen «gesteuerten Modellen» und "Modellen ohne Steuerung» wählen können.

2. Modelle und Versicherungsprodukte der integrierten Versorgung dürfen sich nicht nur durch die nachgewiesenermassen tieferen Behandlungskosten legitimieren. Eine bessere Koordination und Steuerung der Behandlung muss sich im Vergleich zu einer «ungesteuerten Behandlung» auch durch höhere Qualität ausweisen. Dafür sind geeignete Methoden und Massnahmen zu erarbeiten.

3. Für Patienten entscheidend sind das Gesamtresultat (Outcome) und die Sicherheit von Behandlung und Betreuung. Deshalb sollen Anreize entwickelt werden, welche (integrierte) Leistungserbringer motivieren, Outcome- und Performance-Daten konsequent und kontinuierlich zu erheben und auch für Patienten zugänglich zu machen.

\section{Synthese: Voraussetzungen für intelligente Kompromisse}

Unter den Akteuren besteht grundsätzlicher Konsens, dass

- integrierte Versorgung durch eine verbindliche Koordination und Steuerung sowohl ein besseres Kosten-Nutzen-Verhältnis wie eine höhere Qualität der Behandlung ermöglicht;

- Kosteneffektivität und Qualitätsoptimierung vor allem dort zu erwarten sind, wo verbindliche Regeln $\mathrm{zu}$ Koordination und Steuerung zwischen Leistungserbringenden und Versicherern vertraglich vereinbart sind;

- integrierte Versorgung zukünftig vor allem bei komplexen, chronischen Krankheiten und bei Langzeitbetreuten bedeutsam sein wird;

- integrierte Versorgung den elektronischen Zugang und Austausch der Patienteninformationen entlang der Behandlungskette im jeweiligen Betreuungsteam durch den datenschutzkonformen Einsatz von E-Health fördert;

- im Rahmen der Verträge zwischen Versicherern und Ärztenetzen eine selektive Lockerung des Vertragszwangs bereits heute existiert und sinnvoll ist;

- höhere Kosteneffektivität und Qualitätsoptimierung allen Beteiligten nützen: den Leistungserbringenden, den Versicherern und den Patienten;

- Versicherte mit alternativen Versicherungsmodellen finanziell begünstigt werden sollen (Prämienrabatte, differenzierte Selbstbehalte);

- Versorgungs- und Behandlungsqualität (Outcome, Performance) verstärkt evaluiert und zugänglich gemacht werden sollen;

- Versicherte in der Wahl ihres Versicherungsprodukts und Leistungserbringende in der Wahl ihres Versorgungsmodells frei sein sollen;

- die ab 2012 geltende Verfeinerung des Risikoausgleichs richtig ist und allenfalls weiterzuentwickeln ist. 
Ein grundsätzlicher Konsens ist noch zu finden bezüglich der Nichtvertrags- bzw. Listenmodelle: Die Versicherer wollen sie uneingeschränkt anbieten können, die Ärzte lehnen das ab. Dabei ist folgender Kompromiss denkbar: Versicherer bieten nur dort Listenmodelle an, wo keine Vertragsmodelle möglich sind. Bei Vertragsmodellen gilt das Tariffestsetzungsverfahren nach KVG, falls sich die Partner nicht einigen können. Während des Festsetzungsverfahrens werden die AVM unverändert weitergeführt. Dadurch soll wie bei der konventionellen Grundversicherung verhindert werden, dass die betroffenen Versicherten Nachteile in Kauf nehmen müssen, wenn sich die Tarifpartner nicht einigen können.

Integrierte Versorgungsmodelle können nicht von einem Partner des Gesundheitswesens alleine vorgeschlagen und entwickelt werden. Intelligente Kompromisse können nur im Dialog der Partner entwickelt und entscheidungsreif vorbereitet werden. Deshalb braucht es einen «runden Tisch» mit interessierten Teilnehmern: Patienten, Politik (Bund und Kantone), Versicherer, Ärztenetzwerke und Ärzteschaft.

\section{Neue Gesichter in der Redaktion der SÄZ}

\section{Bruno Kesseli, Chefredaktor}

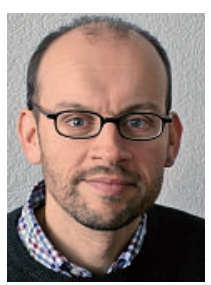

Thomas Heuer

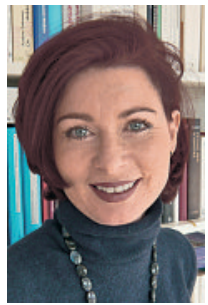

Annette Eichholtz

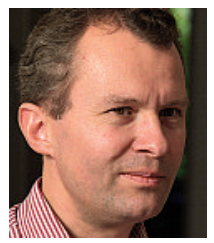

Christoph Rehmann-Sutter

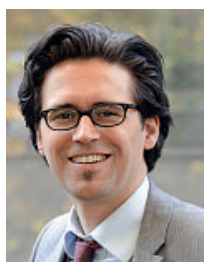

Rouven Porz
Per 1. Juni kam es in der Redaktion der Schweizerischen Ärztezeitung (SÄZ) zu zwei Wechseln, über die nachfolgend kurz informiert wird. Die Stellen von Thomas Heuer und Christoph Rehmann-Sutter wurden durch Annette Eichholtz und Rouven Porz besetzt.

\section{Thomas Heuer: Auf zu neuen Ufern}

Es ist nicht ganz einfach, sich die SÄZ-Redaktion ohne ihn vorzustellen: Während über 11 Jahren war Thomas Heuer als Managing Editor der SÄZ dafür besorgt, dass die Abläufe von der Einreichung der Manuskripte bis zur Produktion der druckfertigen Artikel reibungslos liefen und die Leserinnen und Leser jede Woche eine Zeitschrift in den Händen hielten, die den publizistischen Standards des Schweizerischen Ärzteverlags genügte. Und als Not am Mann war, übernahm er für rund ein halbes Jahr auch das Kommando auf der Brücke und führte die SÄZ sicher durch die chefredaktorlose Zeit von April bis Oktober 2005.

Nun hat sich Thomas Heuer selbständig gemacht und bricht zu neuen Ufern auf. Wir danken unserem liebenswürdigen Kollegen herzlich für die geleisteten Dienste und wünschen ihm eine gute Fahrt in die Zukunft!

Einen Rückblick auf seine 111/2 Jahre bei EMH gibt Thomas Heuer im «Zu guter Letzt» dieser Ausgabe (letzte Heftseite).

\section{Annette Eichholtz: Von «brainstorm» zur SÄZ}

Obwohl mit Thomas Heuer einiges Know-how verloren geht, braucht uns ob seines Weggangs glücklicherweise nicht bange zu werden. Denn mit Annette Eichholtz M.A. hat eine bestens qualifizierte Kollegin mit reicher publizistischer Erfahrung seine Nachfolge als Managing Editor angetreten. Nach dem Studium der Erziehungswissenschaften, Germanistik und Philosophie arbeitete Annette Eichholtz während zwölf Jahren als Verlagslektorin und führte danach die von der Deutschen Hirntumorhilfe herausgegebene neuroonkologische Zeitschrift «brainstorm» acht Jahre lang als Chefredaktorin. Wir heissen die neue Kollegin herzlich willkommen und wünschen ihr einen guten Start!

Annette Eichholtz wird sich der Leserschaft der SÄZ im «Zu guter Letzt» der Nummer 25 vom 17. Juni vorstellen.

\section{Christoph Rehmann-Sutter: \\ Berufung nach Lübeck}

Ein per se erfreuliches Ereignis führte zum bedauerlichen Rücktritt von Christoph Rehmann-Sutter aus der Redaktion Ethik der SÄZ. Die in den Medien bereits breit vermeldete Berufung auf eine Professur für Theorie und Ethik der Biowissenschaften am Institut für Medizin- und Wissenschaftsgeschichte an der Universität Lübeck verlangte von ihm eine Neuausrichtung und Bündelung der Kräfte, die ihn dazu bewog, nicht nur sein Amt als Präsident und Kommissionsmitglied der Nationalen Ethikkommission abzugeben, sondern auch seine Redaktorenstelle bei der SÄZ zur Verfügung zu stellen. Die Redaktion verliert mit Christoph Rehmann-Sutter ein äusserst geschätztes und kompetentes Mitglied, das sich trotz seiner zahlreichen Aufgaben durch stete Ansprechbarkeit auszeichnete und mit kritischen, aber durchwegs konstruktiv-unterstützenden Reviewkommentaren den Autoren eine grosse Hilfe bei der Verbesserung ihrer Arbeiten war. Christoph Rehmann-Sutter wird der Redaktion und der Leserschaft aber insofern erhalten bleiben, als er in der SÄZ auch in Zukunft mit gelegentlichen Beiträgen in Erscheinung treten wird.

Ein ausführliches Interview mit Christoph Rehmann-Sutter erscheint in einer der nächsten Ausgaben der SÄZ.

\section{Rouven Porz: Kontinuität gewährleistet}

Auch im Fall des Rücktritts von Christhoph RehmannSutter gibt es neben einem weinenden auch ein lachendes Auge. Dieses ist auf seinen Nachfolger Rouven Porz gerichtet: Wie Christoph Rehmann-Sutter, bei dem er in Basel seine Dissertation verfasste, ist Rouven Porz sowohl Biologe als auch Philosoph, eine Kombination, die angesichts der zunehmend komplexen ethischen Fragen im Bereich der Biowissenschaften für die Redaktion der SÄZ besonders wertvoll ist. Zudem ist Rouven Porz als Leiter der Ethikstelle des Inselspitals Bern sehr nahe an der klinischen Realität, mit der Ärztinnen und Ärzte täglich konfrontiert sind, was ihm bei seiner Arbeit für die SÄZ ebenfalls zugute kommen dürfte. Die Redaktion heisst auch Rouven Porz herzlich willkommen! Rouven Porz wird sich der Leserschaft der SÄZ ebenfalls in einer der nächsten Ausgaben in der Kolumne «Zu guter Letzt» vorstellen. 